XIII.

(Aus der physiologisch-chemischen Abteilung des städt. Krankenhauses im Friedrichshain in Berlin.)

\title{
Die Organspeicherung des Guajakols und der Brenzkatechinmonoazetsäure.
}

\author{
Von \\ Dr. Jürgens.
}

\begin{abstract}
Hinsichtlich der Resorption und Ausscheidung der Brenzkatechinmonoazetsäure hat Boruttau ${ }^{1}$ ) gefunden, dass ihr Verhalten im Organismus des Hundes, Kaninchens und Menschen demjenigen der Salizylsäure entspricht, der sie insofern nahe steht, als ihre chemische Formel $z u$ derjenigen des Guajakols sich verhält wie diejenige der Salizylsäurc zu der des Phenols: ihre antipyretischen und analgetischen Wirkungen ähneln denjenigen der Azetylsalizylsäure.
\end{abstract}

Die spezifische Wirkung der Salizylpräparate bei Gelenkaffektionen findet nach den Untersuchungen von Bondi und M. Jacoby ${ }^{2}$ ) ihre Begründung in einem besonderen Vermögen der Gelenksubstanz (vermutlich Gelenkknorpel und Synovia), Salizylsäure aufzuspeichern, die beim mit Eitererregern infizierten Organismus noch besonders verstärkt ist. Es lag nahe, die Brenzkatechinmonoazetsäure auch in dieser Hinsicht zu prüfen, ausserdem aber auch ihre Beziehung zum Guajakol hinsichtlich etwaiger Organspeicherung $z u$ berücksichtigen. Bekanntlich ist das Guajakol die wirksame Substanz bei der Kreosotbehandlung der Lungenphthise, und seine Verbindungen werden zur Bekämpfung von entzündlichen Affektionen der Atemwege viel verwendet: in letzterer Beziehung leistet ja auch gerade die Brenzkatechinmonoazetsäure bzw. ihr Kalksalz Vortreffliches.

Ob die Respirationsorgane ein besonderes Speicherungs- bzw. Elektionsvermögen für Guajakol besitzen, ist bis jetzt noch nicht untersucht. Es mussten also in dieser Richtung Versuche angestellt werden, ebenso wie wir es auch für zweckmässig hielten, in einem Vorversuch die Speicherungsverhältnisse der Salizylsäure erneut festzustellen.

Sämtliche Versuche wurden an Kaninchen angestellt, denen die betreffenden Substanzen: Natriumsalizylat gelöst, brenzkatechinmonoazetsaures Kalzium in Wasser verteilt, Guajakol in wenig Alkohol gelöst und mit viel Gummischleim emulgiert, mit der Schlundsonde in den Magen eingeführt wurden, mehrfach in wiederholten kleinen Dosen. Zu geeigneter

1) Zeitschr. f. exp. Path. u. Ther. Bd. 20. S. 298.

2) Hofmeister's Beitr. z. chem. Physiol. u. Path. Bd. 7. S. 514. 
Zeit wurden die Tiere aus einer Karotis entblutet unter gleichzeitiger Linführung isotonischer Salzlösung in eine Jugularvene, bis nur noch ganz wässeriges, stark verdünntes Blut aus der Arteric kam und die Organe blass und blutleer aussahen. Zur Untersuchung verwendet wurden in allen Versuchen: 1. Lunge mit Trachea und Kehlkopf, 2. Nieren, 3. Muskel, 4. Gelenke (beide Kniegelenke mit möglichst wenig Knochenteilen und von Muskelsubstanz möglichst befreit), 5. Blut.

\begin{tabular}{|c|c|c|c|c|c|c|c|c|c|c|c|c|c|}
\hline 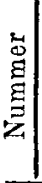 & $\begin{array}{c}\text { Ge- } \\
\text { wicht } \\
8\end{array}$ & Dosis & 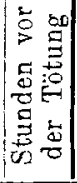 & $\begin{array}{l}\text { Reaktion } \\
\text { für Lunge } \\
\text { und } \\
\text { Trachea }\end{array}$ & 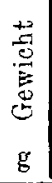 & $\begin{array}{l}\text { Reaktion } \\
\text { für die } \\
\text { Nieren }\end{array}$ & $\begin{array}{l}\stackrel{\overrightarrow{0}}{0} \\
\stackrel{0}{0} \\
0 \\
g\end{array}$ & $\begin{array}{l}\text { Roaktion } \\
\text { für den } \\
\text { Muskel }\end{array}$ & 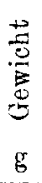 & $\begin{array}{l}\text { Reaktion } \\
\text { für die } \\
\text { Golenke }\end{array}$ & $\begin{array}{l}\frac{+3}{3} \\
0 \\
0 \\
0\end{array}$ & $\begin{array}{c}\text { Reaktion } \\
\text { für das } \\
\text { Blut }\end{array}$ & $\mathrm{cem}$ \\
\hline
\end{tabular}

1. Salizylsaures Natron.

$1|1300| 1 \mathrm{~g} \quad 24 \mid$ stark $|11|$ sehr stark $10 \mid$ schwach $|11|$ deutlich $|22|$ nicht unicrsucht

2. Brenakatechinmonoazetsaures Kalzium.

\begin{tabular}{|c|c|c|c|c|c|c|c|c|c|c|c|c|}
\hline 1 & 1650 & $2 \mathrm{~g}$ & 24 & sehr & 12 & stark & 11 & sehr & 33 & schwach & 22 & stark \\
\hline 2 & 1500 & $2 g$ & 48 & f & & & & SenWals & & & & \\
\hline & & $\begin{array}{l}+2 g \\
+1 g\end{array}$ & $\begin{array}{r}20 \\
2\end{array}$ & $\int$ sebwach & 10 & deutlich & 10 & fehlt & 14 & feblt & 17 & deutlich \\
\hline 3 & $\begin{array}{l}1200 \\
\text { ziert, } \\
\text { gus- } \\
\text { imon. }\end{array}$ & $\begin{array}{r}2 g \\
+1 g\end{array}$ & $\begin{array}{r}24 \\
2\end{array}$ & \} $\begin{array}{c}\text { sehr } \\
\text { deutilich }\end{array}$ & 9 & $\begin{array}{c}\text { sehr } \\
\text { deutlich }\end{array}$ & 10 & fehlt & 20 & deutlich & 15 & nicht unter \\
\hline
\end{tabular}

3. Guajakol.

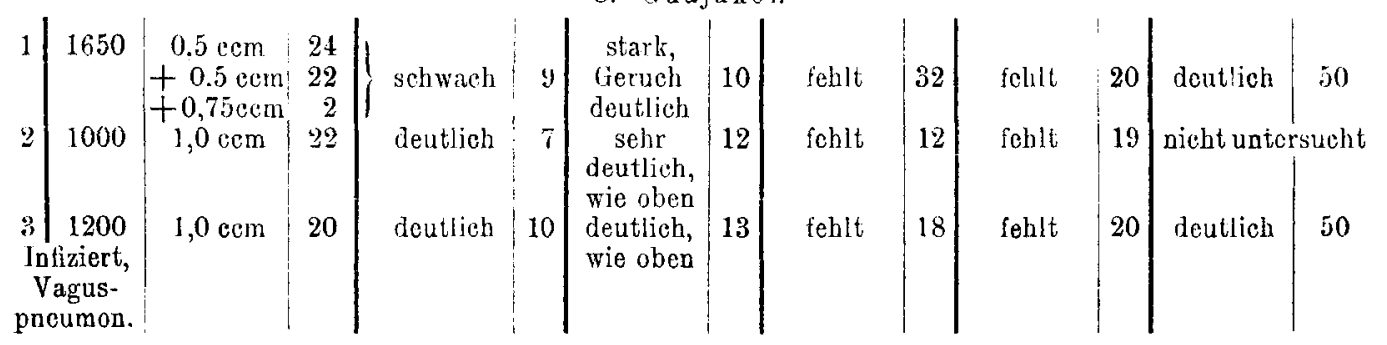

Zur Bestimmung des Gehaltes an Salizylsäure wurde so verfahren, wie es Bondi und Jacoby genau beschrieben haben. Auch zur Bestimmung des Gehaltes an Brenzkatechinmonoazetsäure konnte ebenso verfahren werden, da diese Verbindung sich mit Aether aus den angesäuerten Organextrakten extrahieren lässt und Violettfärbung gibt wie dic Salizylsäure, deren Intensität ein brauchbares Mass für den relativen Gehalt bildet. Zur Bestimmung des Guajakolgehaltes wurde nicht mit verdünnter Schwefelsäure, sondern mit doppelt so starker Salzsäure bzw. mit salzsaurem Alkohol gekocht bzw. extrahiert, um etwa in den Organen oder dem Blut vorhandene Guajakol-Aetherschwefelsäure zu spalten. Die Fällung des letzten Abdampfrückstandes mit Bleiazetat wurde unterlassen; der Aetherextrakt aus demselben auch wieder bei Zimmertemperatur verdunstet, wobei merklicher Guajakolgehalt sich schon am Geruch des Rückstandes kundgab, der mit absolutem Alkohol - je $2 \mathrm{ccm}-$ 
aufgenommen und mit etwas Eisenchloridlösung geprüft wurde, die bei starkem Guajakolgehalt blaugrüne, bei schwächerem graugrünliche Färbung gibt. Bei je einem der Tiere, die brenzkatechinmonoazetsaures Kalzium und Guajakol erhielten, wurde allgemeine Infektion durch Injektion von Staphylokokken in die Bauchhöhle erzeugt und ausserdem durch beiderseitige Vagusdurchschneidung am Halse die Lunge pneumonisch gemacht.

Nebenstehende Tabelle gibt eine Uebersicht über die Ergebnisse.

Es ist folgendes daraus unmittelbar zu ersehen:

1. Ueberall, wo es untersucht wurde, enthält das Blut die betreffende Substanz reichlich. Am nächsten kommt ihr stets die Niere, als Hauptausscheidungsorgan.

2. In dem Salizylsäureversuch enthielten die Gelenke mehr Salizylsäure als dic Muskulatur, womit ihr besonderes Elektionsvermögen dafür bestätigt ist. Aber auch die Atmungsorgane enthielten nicht wenig Salizylsäure. Angesichts der bekannten antikatarrhalischen Wirkung des Aspirins usw. ist dieser Befund bemerkenswert (Bondi und Jacoby hatten die Atmungsorgane nicht in den Kreis ihrer Versuche gezogen).

3. In den Versuchen mit Guajakol enthielten die Atmungsorgane stets diese Substanz, Muskeln und Gelenke niemals.

4. Von den Versuchen mit brenzkatechinmonoazetsaurem Kalzium war in einem in den Gelenken keine Spur der Substanz nachweisbar, in zwei anderen, darunter einem mit allgemeiner Infektion, war die Reaktion nschwach". Die Atmungsorgane enthielten in allen Fällen Brenzkatechinmonoazetsäure.

Es darf also aus unseren Versuchsergebnissen gefolgert werden, dass die Atmungsorgane ein besonderes Elektions- bzw. Speicherungsvermögen für Guajakol besitzen, ebenso für die Brenzkatechinmonoazetsäure in erheblichem Masse, wogegen die Speicherung in den Gelenken hier geringer ist. 